\title{
Axially chiral benzimidazolium based silver(I) and gold(I) bis-NHC complexes of R-BINOL scaffold: synthesis, characterization and DFT studies
}

\author{
SONALI RAMGOPAL MAHULE* \\ Department of Chemistry, Indian Institute of Technology Bombay, Powai, Mumbai, Maharashtra 400 076, India \\ E-mail: sonalimahule@chem.iitb.ac.in
}

MS received 11 May 2017; revised 8 July 2017; accepted 18 July 2017; published online 4 September 2017

\begin{abstract}
The axially chiral ligand of $R$-BINOL scaffold was synthesized by a series of manipulations which involved different chemical reactions to obtain the desired sliver(I) and gold(I) $\left\{\left[\mathrm{L}\left(\mathrm{L}^{\prime}-\mathrm{NHC}\right)_{2}\right] \mathrm{M}\right\} \mathrm{Cl}$ $\left(\mathrm{L}=3,3^{\prime}\right.$-dimethyl-2,2'-dimethoxy-1,1'-binaphthyl, $\mathrm{L}^{\prime}=i$-propyl-benzo[d]imidazole $)(\mathrm{M}=\mathrm{Ag}$ and $\mathrm{Au})$ complexes. Enantiopure $R$-BINOL was employed as a basic unit to synthesize a benzimidazole based bisNHC ligand $\mathbf{1 g}$ which was obtained through the formation of different intermediate $\mathbf{1}(\mathbf{a}-\mathbf{f})$ compounds. The newly synthesized bis-NHC ligand precursor (1g) and its corresponding $\left\{\left[\mathrm{L}^{\prime}\left(\mathrm{L}^{\prime}-\mathrm{NHC}\right)_{2}\right] \mathrm{Ag}\right\} \mathrm{Cl}(\mathbf{1 h})$ and $\left\{\left[\mathrm{L}\left(\mathrm{L}^{\prime}-\mathrm{NHC}\right)_{2}\right] \mathrm{Au}\right\} \mathrm{Cl}(\mathbf{1 i})$ complexes were characterized by different spectroscopic techniques. The geometries of the optimized structure of the complexes $\mathbf{1 h}$ and $\mathbf{1 i}$ were computed at the B3LYP/SDD, 6-31G(d) level. Low temperature fluorescence spectroscopic studies did not show any evidence for the weak metal-metal interaction in these complexes.
\end{abstract}

Keywords. Chiral bis-N-heterocyclic carbene ligand; $\mathrm{Ag}(\mathrm{I})$ complex; $\mathrm{Au}(\mathrm{I})$ complex; computational study; electronic property.

\section{Introduction}

The development of chiral N-heterocyclic carbene (NHC) has been a very exciting topic in coordination chemistry for the last two decades. This trend is expanding day by day but, it is less likely to be explored in other interesting areas for their applications beyond the asymmetric catalysis. ${ }^{1-7}$ At the same time, achiral NHCs have widely been studied for their different properties $^{8-11}$ and applications in the fields of medicine for drug designing, ${ }^{12-14}$ nanosciences ${ }^{15,16}$ and materials. ${ }^{17}$ Herein, we developed a new benzimidazole based chiral bis-N-heterocyclic carbene ligand (1g) and its silver(I) (1h) and gold(I) (1i) complexes for their future perspective of studying their properties and applications.

A phenomenal progress has been made in synthesis, characterization and reactions of NHCs with unique electronic and steric properties. The structures can easily be modified by change in functional group in imidazolium ring, principally at the nitrogen, with different

\footnotetext{
*For correspondence
}

organic and inorganic moieties. ${ }^{18-22}$ The functionalization of the NHC ligand can be utilized in different ways depending upon the metal and also on the oxidation state of the metal coordinated to NHC ligand. ${ }^{23}$ Therefore, the chemistry of NHCs has become mainstream in organometallics, challenging the popular tert-phosphine ligands. ${ }^{18,24-26}$ However, the importance of optically active transition metal complexes of NHC ligands is interestingly increasing because of the current attraction in the preparation of new optical devices. ${ }^{27}$

Furthermore, in asymmetric catalysis, N-heterocyclic carbene ligands are popular as a growing class of ligands that can be a substituent to a phosphine ligand and produce more efficient metal complexes owing to their stability to air and moisture with strong $\sigma$ donar and poor $\pi$-acceptor properties. ${ }^{28,29}$ In light of these facts, many $\mathrm{N}$-heterocyclic carbene ligands are designed with different structural motifs, coordinated with various transition metals like copper(I), silver(I), gold(I), mercury(II), palladium(II), rhodium(III), ruthenium(II), etc., and are employed not only for various organometallics ${ }^{30,31}$ but for organocatalytic

Electronic supplementary material: The online version of this article (doi:10.1007/s12039-017-1352-9) contains supplementary material, which is available to authorized users. 
transformations. ${ }^{32}$ Herein, we report and discuss one such versatile chiral structural motif, BINOL and its benzimidazole based N-heterocyclic carbene complexes. $R$ and $S$ enantiomers of BINOL have been extensively used as chiral ligands in a number of asymmetric reactions. ${ }^{33}$ Their chirality is based on the blocked rotation around the $\mathrm{C}-\mathrm{C}$ axis linking the two napthyl units giving configurationally stable atropisomers. ${ }^{34}$ Excellence in enantioselectivity of BINOL-type chiral $\mathrm{N}$-heterocyclic ligand is its inflexible backbone and rigid chiral pocket, which allow only one face of substrate for attack and binding. The binapthyl backbone imposes $\mathrm{C}_{2}$ symmetry upon the bis-carbene ligand and mutual antiorientation of $\mathrm{N}$-alkyl substituent with respect to the plane into which chelate ring is inscribed. ${ }^{35}$

However, a very fascinating interaction between the closed shells of many organometallic or inorganic compounds of $d^{2}, d^{8}$ and $d^{10}$ systems are interestingly being recognized as important determinants of solid-state structures as well as potential sources of useful materials. ${ }^{36-38}$ These closed shell contacts are commonly known as metallophilic interactions and are experimentally observed by X-ray diffraction studies and by the electronic properties of complexes in its solid state.
In this work, we studied the electronic properties of $\mathrm{Ag}(\mathrm{I})$ and $\mathrm{Au}(\mathrm{I})$ complexes to check such M-M interactions in the solid state structure of the complexes of $\left\{\left[\mathrm{L}\left(\mathrm{L}^{\prime}-\mathrm{NHC}\right)_{2}\right] \mathrm{M}\right\} \mathrm{Cl}$ type.

\section{Experimental}

\subsection{General procedures}

All manipulations were carried out using standard Schlenk techniques. Solvents were purified by standard procedures. $R$-BINOL and $n$-BuLi were purchased from Spectrochem (India) and used without any further purification. The compounds, viz., 1(a-f) ${ }^{39}$ (see Scheme 1) were synthesized by manipulations of the procedures, reported in the literature. ${ }^{1} \mathrm{H}$ and ${ }^{13} \mathrm{C}\{1 \mathrm{H}\}$ NMR spectra were recorded in $\mathrm{CDCl}_{3}$, DMSO- $d_{6}$, and $\mathrm{CD}_{3} \mathrm{OD}$ on a Bruker 400 and $500 \mathrm{MHz}$ NMR spectrometers. ${ }^{1} \mathrm{H}$ NMR peaks are labelled as singlet (s), doublet (d), triplet (t), triplet of doublets (td) and multiplet (m). Infrared spectra were recorded on a Perkin Elmer Spectrum One FT-IR spectrometer. Mass spectrometry measurements were done on a Micromass Q-Tof spectrometer and Bruker maxis impact spectrometer. Elemental analysis was carried out on Thermo Finnigan Flash EA 112 SERIES (CHNS) Elemental analyser. Specific optical rotation experiments

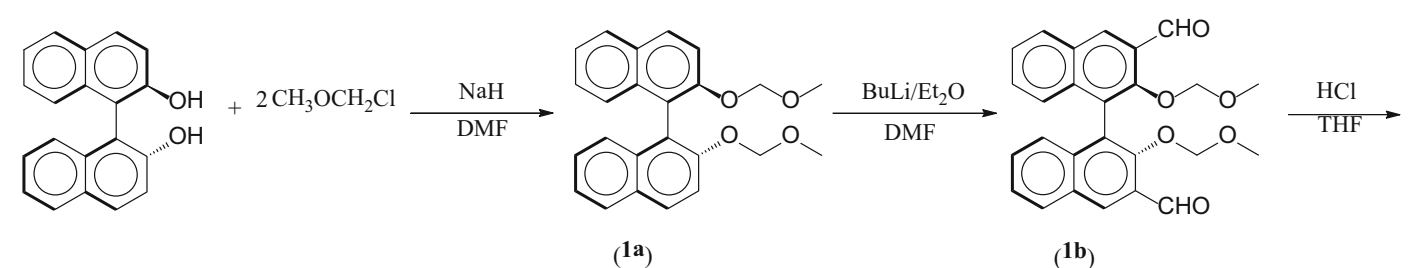

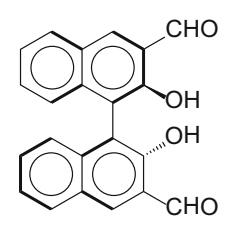

(1c)

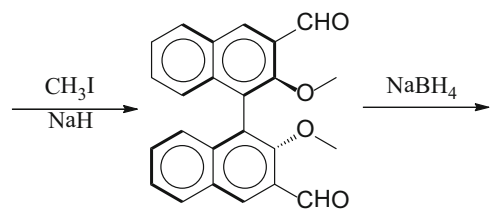

(1d)

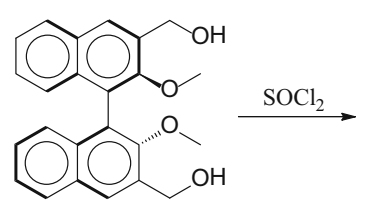

(1e)

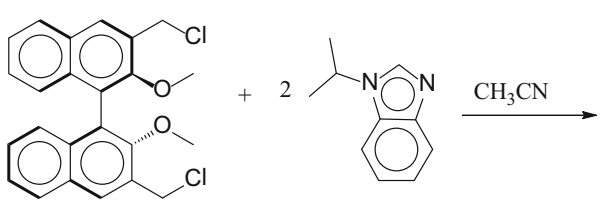

(1f)

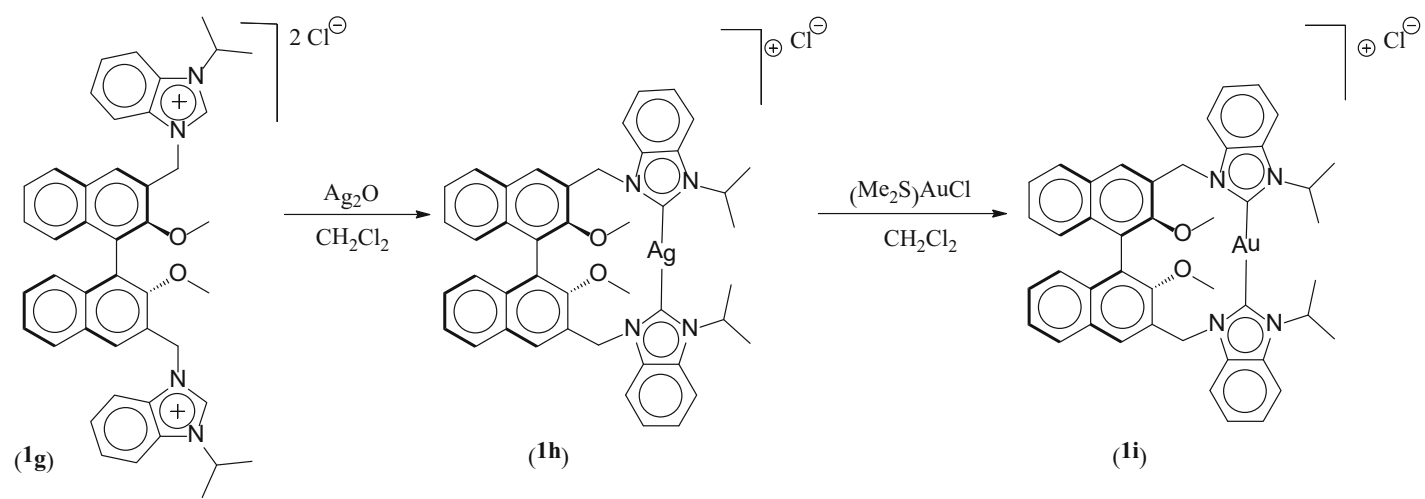

Scheme 1. Synthetic pathway for the $R$-BINOL based axially chiral silver(I) and gold(I) complexes. 
were performed with Autopol IV, Serial \#82083 polarimeter. Absorption spectra were recorded on a Varian UV-Vis spectrophotometer in the range of $200-800 \mathrm{~nm}$ at room temperature whereas fluorescence studies were performed at 77 $\mathrm{K}$ using Horiba Fluoromax-4 spectrophotometer in a glassy solution of EtOH: $\mathrm{CHCl}_{3}(4: 1, \mathrm{v} / \mathrm{v})$.

\subsection{Synthesis of (R)-1,1'-(2,2'-dimethoxy-1,1'-} binaphthyl-3,3'-diyl)bis(methylene)bis(3-i-propylbenzo[d]imidazol-1-ium) chloride

(1g)

A mixture of (R)-3,3'-bis(chloromethyl)-2,2' -dimethoxy-1, $1^{\prime}$ -binaphthyl (1f) $(0.500 \mathrm{~g}, 1.21 \mathrm{mmol})$ and $1-i$-propylbenzo[d]imidazole $(0.48 \mathrm{~g}, 3.04 \mathrm{mmol})$ was refluxed in $\mathrm{CH}_{3} \mathrm{CN}$ (ca. $20 \mathrm{~mL}$ ) for one day, after which the solvent was evaporated in vacuo. The product was purified by column chromatography using silica gel as a stationary phase eluted with $\mathrm{CH}_{2} \mathrm{Cl}_{2}: \mathrm{MeOH}(5: 1 \mathrm{v} / \mathrm{v})$ to give the product $1 \mathrm{~g}(0.473 \mathrm{~g}, 53 \%)$ as a white solid. ${ }^{1} \mathrm{H} \mathrm{NMR}\left(\mathrm{CDCl}_{3}, 500\right.$ $\left.\mathrm{MHz}, 25^{\circ} \mathrm{C}\right): \delta 9.81\left(\mathrm{~s}, 2 \mathrm{H}, \mathrm{NHCHN}\right.$ of $\left.\mathrm{C}_{7} \mathrm{H}_{5} \mathrm{~N}_{2}\right), 8.22(\mathrm{~s}$, $\left.2 \mathrm{H}, \mathrm{C}_{10} \underline{H}_{5}\right), 8.00\left(\mathrm{~d}, 2 \mathrm{H},{ }^{3} J_{\mathrm{HH}}=8 \mathrm{~Hz}, \mathrm{C}_{10} \underline{H}_{5}\right), 7.93(\mathrm{~d}$, $\left.2 \mathrm{H},{ }^{3} J_{\mathrm{HH}}=8 \mathrm{~Hz}, \mathrm{C}_{7} \underline{H}_{5} \mathrm{~N}_{2}\right), 7.88\left(\mathrm{~d}, 2 \mathrm{H},{ }^{3} J_{\mathrm{HH}}=8 \mathrm{~Hz}\right.$, $\left.\mathrm{C}_{7} \underline{H}_{5} \mathrm{~N}_{2}\right), 7.63\left(\mathrm{t}, 2 \mathrm{H},{ }^{3} J_{\mathrm{HH}}=8 \mathrm{~Hz}, \mathrm{C}_{7} \underline{H}_{5} \mathrm{~N}_{2}\right), 7.54(\mathrm{t}$, $\left.2 \mathrm{H},{ }^{3} J_{\mathrm{HH}}=8 \mathrm{~Hz}, \mathrm{C}_{7} \underline{H}_{5} \mathrm{~N} 2\right), 7.38\left(\mathrm{t}, 2 \mathrm{H},{ }^{3} J_{\mathrm{HH}}=8 \mathrm{~Hz}\right.$, $\left.\mathrm{C}_{10} \underline{H}_{5}\right), 7.20\left(\mathrm{t}, 2 \mathrm{H},{ }^{3} \mathrm{~J}_{\mathrm{HH}}=8 \mathrm{~Hz}, \mathrm{C}_{10} \underline{H}_{5}\right), 6.96(\mathrm{~d}, 2 \mathrm{H}$, $\left.{ }^{3} J_{\mathrm{HH}}=8 \mathrm{~Hz}, \mathrm{C}_{10} \underline{H}_{5}\right), 5.98\left(\mathrm{~d}, 2 \mathrm{H},{ }^{2} J_{\mathrm{HH}}=15 \mathrm{~Hz}, \underline{\mathrm{CH}}_{2}\right)$, $5.92\left(\mathrm{~d}, 2 \mathrm{H},{ }^{2} J_{\mathrm{HH}}=15 \mathrm{~Hz}, \underline{\mathrm{H}}_{2}\right), 5.05$ (sept, $2 \mathrm{H},{ }^{3} J_{\mathrm{HH}}=6$ $\left.\mathrm{Hz}, \mathrm{CH}\left(\mathrm{CH}_{3}\right)_{2}\right), 3.00\left(\mathrm{~s}, 6 \mathrm{H}, \mathrm{CH}_{3}\right), 1.66\left(\mathrm{~d}, 12 \mathrm{H},{ }^{3} J_{\mathrm{HH}}=\right.$ $\left.6 \mathrm{~Hz}, \mathrm{CH}\left(\mathrm{C}_{3}\right)_{2}\right) .{ }^{13} \mathrm{C}\left\{{ }^{1} \mathrm{H}\right\} \mathrm{NMR}\left(\mathrm{CD}_{3} \mathrm{OD}, 125 \mathrm{MHz}\right.$, $\left.25^{\circ} \mathrm{C}\right): \delta 155.6\left(\underline{C}_{10} \mathrm{H}_{5}\right), 141.8\left(\underline{C}_{7} \mathrm{H}_{5} \mathrm{~N}_{2}\right), 135.8\left(\underline{C}_{7} \mathrm{H}_{5} \mathrm{~N}_{2}\right)$, $132.9\left(\underline{C}_{7} \mathrm{H}_{5} \mathrm{~N}_{2}\right), 132.8\left(\underline{C}_{10} \mathrm{H}_{5}\right), 132.4\left(\underline{C}_{10} \mathrm{H}_{5}\right), 131.7$ $\left(\underline{C}_{10} \mathrm{H}_{5}\right), 129.7\left(\underline{C}_{10} \mathrm{H}_{5}\right), 128.7\left(\underline{C}_{10} \mathrm{H}_{5}\right), 128.2\left(\underline{C}_{10} \mathrm{H}_{5}\right)$, $128.1\left(\underline{C}_{10} \mathrm{H}_{5}\right), 127.7\left(\underline{C}_{10} \mathrm{H}_{5}\right), 126.8\left(\underline{C}_{7} \mathrm{H}_{5} \mathrm{~N}_{2}\right), 126.3$ $\left(\underline{C}_{7} \mathrm{H}_{5} \mathrm{~N}_{2}\right), 125.2\left(\underline{C}_{10} \mathrm{H}_{5}\right), 115.1\left(\underline{C}_{7} \mathrm{H}_{5} \mathrm{~N}_{2}\right), 115.05\left(\underline{C}_{7} \mathrm{H}_{5}\right.$ $\left.\mathrm{N}_{2}\right), 61.3\left(\underline{C H}_{2}\right), 52.7\left(\underline{C H}\left(\mathrm{CH}_{3}\right)_{2}\right), 48.8\left(\underline{C H}_{3}\right), 22.3$ $\left(\mathrm{CH}\left(\mathrm{CH}_{3}\right)_{2}\right), 22.2\left(\mathrm{CH}\left(\underline{C H}_{3}\right)_{2}\right)$. HRMS (ES): $m / z 330.1725$ $[\mathrm{M}-2 \mathrm{Cl}]^{2+}$ calcd. 330.1727. IR data in $\mathrm{KBr}$ pellet, $\nu / \mathrm{cm}^{-1}$ : 2979 (s), 1621 (m), 1557 (s), 1499 (m), 1318 (m), 1242 (s), 1206 (s), 1147 (m), 1097 (s), 1039 (m), 1002 (m), 893 (m), 798 (m), 750 (s), 496 (m). Anal. Calc. for $\mathrm{C}_{44} \mathrm{H}_{44} \mathrm{Cl}_{2} \mathrm{~N}_{4} \mathrm{O}_{2} \bullet$ $\mathrm{CHCl}_{3}$ : C, 63.50; H, 5.33; N, 6.58\%. Found: C, 62.33; H, 5.89; N, $7.54 \%$.

\subsection{Synthesis of $\left[(R)-1,1^{\prime}-\left(2,2^{\prime}\right.\right.$-dimethoxy-1,1'-} binaphthyl-3,3'-diyl)bis(methylene)bis(3-i-propylbenzo[d]imidazol-2-ylidene) $\mathrm{Ag}][\mathrm{Cl}]$

(1h)

A mixture of $(R)-1,1^{\prime}$-(2,2' -dimethoxy-1, $1^{\prime}$-binaphthyl-3,3'diyl)bis(methylene) bis (3-isopropyl-benzo[d]imidazol-1-ium) chloride (1g) (0.250 g, $0.340 \mathrm{mmol})$ and $\mathrm{Ag}_{2} \mathrm{O}(0.078 \mathrm{~g}, 0.340$ $\mathrm{mmol}$ ) in $\mathrm{CH}_{2} \mathrm{Cl}_{2}$ (ca. $10 \mathrm{~mL}$ ) was stirred at room temperature in dark for overnight. The reaction mixture was filtered through a pad of celite to remove excess inorganic salt and then the solvent was removed in vacuo to obtain the product 1h as a white solid $(0.256 \mathrm{~g}, 93 \%) .{ }^{1} \mathrm{H}$ NMR $\left(\mathrm{CDCl}_{3}, 500\right.$ $\left.\mathrm{MHz}, 25^{\circ} \mathrm{C}\right): \delta 7.75\left(\mathrm{~d}, 1 \mathrm{H},{ }^{3} \mathrm{~J}_{\mathrm{HH}}=7 \mathrm{~Hz}, \mathrm{C}_{7} \underline{H}_{4} \mathrm{~N}_{2}\right), 7.65$ $\left(\mathrm{d}, 2 \mathrm{H},{ }^{3} J_{\mathrm{HH}}=8 \mathrm{~Hz}, \mathrm{C}_{10} \mathrm{H}_{4}\right), 7.55\left(\mathrm{~d}, 2 \mathrm{H},{ }^{3} J_{\mathrm{HH}}=8\right.$ $\left.\mathrm{Hz}, \mathrm{C}_{7} \underline{H}_{4} \mathrm{~N}_{2}\right), 7.38\left(\mathrm{~d}, 2 \mathrm{H},{ }^{3} \mathrm{~J}_{\mathrm{HH}}=8 \mathrm{~Hz}, \mathrm{C}_{7} \underline{H}_{4} \mathrm{~N}_{2}\right), 7.35$ $\left(\mathrm{d}, 2 \mathrm{H},{ }^{3} J_{\mathrm{HH}}=8 \mathrm{~Hz}, \mathrm{C}_{7} \underline{H}_{4} \mathrm{~N}_{2}\right), 7.32\left(\mathrm{t}, 2 \mathrm{H},{ }^{3} J_{\mathrm{HH}}=8\right.$ $\left.\mathrm{Hz}, \mathrm{C}_{10} \underline{H}_{5}\right), 7.25\left(\mathrm{t}, 2 \mathrm{H},{ }^{3} J_{\mathrm{HH}}=8 \mathrm{~Hz}, \mathrm{C}_{10} \underline{H}_{5}\right), 7.16(\mathrm{~d}$, $\left.2 \mathrm{H},{ }^{3} J_{\mathrm{HH}}=8 \mathrm{~Hz}, \mathrm{C}_{10} \underline{H}_{5}\right), 5.89\left(\mathrm{~s}, 2 \mathrm{H}, \underline{\mathrm{C}}_{2}\right), 5.16$ (sept, $\left.2 \mathrm{H},{ }^{3} J_{\mathrm{HH}}=7 \mathrm{~Hz}, \mathrm{C} \underline{H}\left(\mathrm{CH}_{3}\right)_{2}\right), 3.20\left(\mathrm{~s}, 6 \mathrm{H}, \underline{\mathrm{C}}_{3}\right), 1.79(\mathrm{~d}$, $\left.12 \mathrm{H},{ }^{3} J_{\mathrm{HH}}=7 \mathrm{~Hz}, \mathrm{CH}\left(\mathrm{C}_{3}\right)_{2}\right) \cdot{ }^{13} \mathrm{C}\left\{{ }^{1} \mathrm{H}\right\} \mathrm{NMR}\left(\mathrm{CDCl}_{3}, 125\right.$ $\left.\mathrm{MHz}, 25^{\circ} \mathrm{C}\right): \delta 187.3(\mathrm{Ag}-\underline{C}), 153.9\left(\underline{C}_{10} \mathrm{H}_{5}\right), 134.3\left(\underline{C}_{10} \mathrm{H}_{5}\right)$, $134.1\left(\underline{C}_{7} \mathrm{H}_{4} \mathrm{~N}_{2}\right), 132.7\left(\underline{C}_{7} \mathrm{H}_{5} \mathrm{~N}_{2}\right), 130.2\left(\underline{C}_{10} \mathrm{H}_{5}\right), 128.9$ $\left(\underline{C}_{10} \mathrm{H}_{5}\right), 128.5\left(\underline{C}_{10} \mathrm{H}_{5}\right), 128.2\left(\underline{C}_{10} \mathrm{H}_{5}\right), 127.3\left(\underline{C}_{10} \mathrm{H}_{5}\right)$, $125.6\left(\underline{C}_{7} \mathrm{H}_{4} \mathrm{~N}_{2}\right), 125.4\left(\underline{C}_{7} \mathrm{H}_{4} \mathrm{~N}_{2}\right), 124.3\left(\underline{C}_{10} \mathrm{H}_{5}\right), 124.2$ $\left(\underline{C}_{10} \mathrm{H}_{5}\right), 124.0\left(\underline{C}_{10} \mathrm{H}_{5}\right), 112.6\left(\underline{C}_{7} \mathrm{H}_{4} \mathrm{~N}_{2}\right), 112.5\left(\underline{C}_{7} \mathrm{H}_{5} \mathrm{~N}_{2}\right)$, $61.5\left(\underline{\mathrm{C}} \mathrm{H}_{2}\right), 53.9\left(\underline{\mathrm{C}} \mathrm{H}\left(\mathrm{CH}_{3}\right)_{2}\right), 48.9\left(\underline{\mathrm{C}} \mathrm{H}_{3}\right), 22.7(\mathrm{CH}$ $\left.\left(\mathrm{CH}_{3}\right)_{2}\right)$. HRMS (ES): $m / z 766.2433$ [M-Cl] $^{+}$calcd. 766.2432. IR data in $\mathrm{KBr}$ pellet, $v / \mathrm{cm}^{-1}: 3056(\mathrm{~m}), 2973(\mathrm{~m})$, $2934(\mathrm{~m}), 1671(\mathrm{~m}), 1622(\mathrm{~m}), 1597(\mathrm{~m}), 1498(\mathrm{~m}), 1476(\mathrm{~s})$, 1387 (s), 1240 (s), 1147 (m), $1088(\mathrm{~m}), 1006(\mathrm{~m}), 886(\mathrm{~m})$, $746(\mathrm{~s}), 668(\mathrm{~m}), 527(\mathrm{~m})$. Anal. Calc. for $\mathrm{C}_{44} \mathrm{H}_{42} \mathrm{AgClN}_{4} \mathrm{O}_{2}$ : C, 65.88; H, 5.28; N, 6.98\%. Found: C, 66.80; H, 5.08; N, $7.55 \%$.

\subsection{Synthesis of $\left[(R)-1,1^{\prime}-\left(2,2^{\prime}\right.\right.$-dimethoxy-1, $1^{\prime}$ - binaphthyl-3,3'-diyl)bis(methylene)bis(3-i-propyl- benzo[d]imidazol-2-ylidene)Au] [Cl] (1i)}

A mixture of $\left[(R)-1,1^{\prime}\right.$ - $\left(2,2^{\prime}\right.$-dimethoxy-1, $1^{\prime}$-binaphthyl-3,3'diyl) bis(methylene) bis(3-i-propyl-benzo[d] imidazol-2-ylidene $) \mathrm{Ag}][\mathrm{Cl}](\mathbf{1 h})(0.200 \mathrm{~g}, 0.249 \mathrm{mmol})$ and $\mathrm{Au}\left(\mathrm{SMe}_{2}\right) \mathrm{Cl}$ $(0.073 \mathrm{~g}, 0.244 \mathrm{mmol})$ in $\mathrm{CH}_{2} \mathrm{Cl}_{2}(c a .10 \mathrm{~mL})$ was stirred in dark at room temperature for overnight. The reaction mixture was filtered through a pad of celite to remove excess inorganic salt and then the solvent was removed in vacuo to obtain a crude product which was purified by column chromatography using silica gel as a stationary phase and eluted with $\mathrm{CH}_{2} \mathrm{Cl}_{2}: \mathrm{MeOH}(99: 1 \mathrm{v} / \mathrm{v})$ to give a product $\mathbf{1 i}$ as white powder $(0.093 \mathrm{~g}, 42 \%) .{ }^{1} \mathrm{H} \mathrm{NMR}\left(\mathrm{CDCl}_{3}, 500 \mathrm{MHz}, 25^{\circ} \mathrm{C}\right)$ : $\delta 7.78\left(\mathrm{~d}, 2 \mathrm{H},{ }^{3} J_{\mathrm{HH}}=7 \mathrm{~Hz}, \mathrm{C}_{7} \underline{H}_{4} \mathrm{~N}_{2}\right), 7.75\left(\mathrm{~d}, 2 \mathrm{H},{ }^{3} J_{\mathrm{HH}}=8\right.$ $\left.\mathrm{Hz}, \mathrm{C}_{10} \underline{H}_{5}\right), 7.68\left(\mathrm{~d}, 2 \mathrm{H},{ }^{3} J_{\mathrm{HH}}=8 \mathrm{~Hz}, \mathrm{C}_{10} \underline{H}_{5}\right), 7.58(\mathrm{~d}$, $\left.2 \mathrm{H},{ }^{3} J_{\mathrm{HH}}=8 \mathrm{~Hz}, \mathrm{C}_{7} \underline{H}_{4} \mathrm{~N}_{2}\right), 7.340\left(\mathrm{t}, 2 \mathrm{H},{ }^{3} J_{\mathrm{HH}}=6\right.$ $\left.\mathrm{Hz}, \mathrm{C}_{7} \underline{H}_{4} \mathrm{~N}_{2}\right), 7.36\left(\mathrm{t}, 2 \mathrm{H},{ }^{3} J_{\mathrm{HH}}=6 \mathrm{~Hz}, \mathrm{C}_{7} \underline{H}_{4} \mathrm{~N}_{2}\right), 7.32$ $\left(\mathrm{t}, 2 \mathrm{H},{ }^{3} J_{\mathrm{HH}}=7 \mathrm{~Hz}, \mathrm{C}_{10} \underline{H}_{5}\right), 7.26\left(\mathrm{t}, 2 \mathrm{H},{ }^{3} J_{\mathrm{HH}}=7\right.$ $\left.\mathrm{Hz}, \mathrm{C}_{10} \underline{H}_{5}\right), 7.16\left(\mathrm{~d}, 2 \mathrm{H},{ }^{3} J_{\mathrm{HH}}=8 \mathrm{~Hz}, \mathrm{C}_{10} \underline{H}_{5}\right) 6.12(\mathrm{~d}$, $\left.2 \mathrm{H},{ }^{2} J_{\mathrm{HH}}=16 \mathrm{~Hz}, \mathrm{CH}_{2}\right), 5.98\left(\mathrm{~d}, 2 \mathrm{H},{ }^{2} J_{\mathrm{HH}}=16 \mathrm{~Hz}\right.$, $\left.\mathrm{C} \underline{H}_{2}\right), 5.60$ (sept, $\left.2 \mathrm{H},{ }^{3} J_{\mathrm{HH}}=7 \mathrm{~Hz}, \mathrm{C} \underline{H}\left(\mathrm{CH}_{3}\right)_{2}\right), 3.28$ $\left(\mathrm{s}, 6 \mathrm{H}, \mathrm{C} \underline{H}_{3}\right), 1.83\left(\mathrm{~d}, 12 \mathrm{H},{ }^{3} J_{\mathrm{HH}}=7 \mathrm{~Hz}, \mathrm{CH}\left(\mathrm{C}_{3}\right)_{2}\right)$. $\left.{ }^{13} \mathrm{C}^{1}{ }^{1} \mathrm{H}\right\} \mathrm{NMR}\left(\mathrm{CDCl}_{3}, 100 \mathrm{MHz}, 25^{\circ} \mathrm{C}\right): \delta 177.9(\mathrm{Au}-\mathrm{C})$, $154.0\left(\underline{C}_{10} \mathrm{H}_{5}\right), 134.2\left(\underline{C}_{10} \mathrm{H}_{5}\right), 134.0\left(\underline{C}_{7} \mathrm{H}_{4} \mathrm{~N}_{2}\right), 132.0$ $\left(\underline{C}_{7} \mathrm{H}_{5} \mathrm{~N}_{2}\right), 130.4\left(\underline{C}_{10} \mathrm{H}_{5}\right), 129.1\left(\underline{C}_{10} \mathrm{H}_{5}\right), 128.4\left(\underline{C}_{10} \mathrm{H}_{5}\right)$, $128.2\left(\underline{C}_{10} \mathrm{H}_{5}\right), 127.4\left(\underline{C}_{10} \mathrm{H}_{5}\right), 125.7\left(\underline{C}_{7} \mathrm{H}_{4} \mathrm{~N}_{2}\right), 125.6$ $\left(\underline{C}_{7} \mathrm{H}_{4} \mathrm{~N}_{2}\right), 124.7\left(\underline{C}_{10} \mathrm{H}_{5}\right), 124.4\left(\underline{C}_{7} \mathrm{H}_{4} \mathrm{~N}_{2}\right), 112.9\left(\underline{C}_{10} \mathrm{H}_{5}\right)$, $112.9\left(\underline{C}_{10} \mathrm{H}_{5}\right), 112.8\left(\underline{C}_{7} \mathrm{H}_{5} \mathrm{~N}_{2}\right), 61.5\left(\underline{C}_{2}\right), 54.5(\underline{C} \mathrm{H}$ $\left.\left(\mathrm{CH}_{3}\right)_{2}\right), 48.1\left(\mathrm{CH}_{3}\right), 22.0\left(\mathrm{CH}\left(\mathrm{CH}_{3}\right)_{2}\right)$. HRMS (ES): $\mathrm{m} / z$

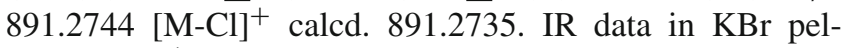
let, $v / \mathrm{cm}^{-1}: 2927(\mathrm{~m}), 1623(\mathrm{~m}), 1438$ (s), 1399 (s), 
1241 (m), 1091 (m), 1007 (m), 747 (s). Anal. Calc. for $\mathrm{C}_{44} \mathrm{H}_{42} \mathrm{AuClN}_{4} \mathrm{O}_{2}$ : C, 59.30; H, 4.75; N, 6.29\%. Found: C, $58.59 ; \mathrm{H}, 4.32 ; \mathrm{N}, 6.58 \%$.

\subsection{Computational Methods}

Density functional theory (DFT) calculations were performed on all the metal complexes $\mathbf{1 h}$ and $\mathbf{1 i}$ using GAUSSIAN $09^{40}$ suite of quantum chemical programs. The Becke three parameter exchange functional in conjunction with Lee-YangParr correlation functional (B3LYP) was employed in the study. ${ }^{41,42}$ The polarized basis set $6-31 \mathrm{G}(\mathrm{d})^{43-45}$ was used to describe chlorine, oxygen, carbon, nitrogen and hydrogen atoms. The Stuttgart-Dresden effective core potential (ECP) along with valence basis sets (SDD) was used for the silver, ${ }^{46-48}$ and gold ${ }^{49,50}$ atoms. Frequency calculations were performed for both the optimized structures to characterize the stationary points as minima.

\section{Results and Discussion}

New axially chiral bis-NHC ligand of $R$-BINOL framework was efficiently synthesized in overall yield of $c a .87 \%$ and was characterized unambiguously by NMR, IR, mass, elemental analysis and polarimetry (Scheme 1). Starting from the commercially available $R$-BINOL, to prepare the ligand, different precursors 1(a-f) (Scheme 1) were synthesized which involved a series of manipulations in the procedures which were reported in the literature. ${ }^{39}$ The straightforward process for the synthesis of ligand precursor $1 \mathrm{~g}$ (Scheme 1) started from the protection of phenolic hydroxyl group of BINOL with the chloromethyl methyl ether obtained (R)-2,2'-bis(methoxymethoxy)$1,1^{\prime}$-binaphthyl (1a) in quantitative yields. The compound 1a on formylation in the presence of $n$-BuLi and DMF afforded $(R)-2,2^{\prime}$-bis(methoxymethoxy)-1,1'binaphthyl-3,3'-dicarbaldehyde (1b) which was then subjected to deprotection and again protection with methyl iodide gave $(R)$-2,2'-dihydroxy-1, $1^{\prime}$-binaphthyl3,3'-dicarbaldehyde (1c) and $(R)$-2,2'-dimethoxy-1,1'binaphthyl-3,3'-dicarbaldehyde (1d), respectively. The reduction of 1d with $\mathrm{NaBH}_{4}$ gave 1e, which was followed by chlorination with $\mathrm{SOCl}_{2}$, which gave $(R)-3,3^{\prime}$ bis(chloromethyl)-2,2'-dimethoxy-1,1'-binaphthyl (1f). The desired compound $\mathbf{1 g}$ was finally obtained by refluxing $\mathbf{1 f}$ and $1-i$-propyl-benzo[d]imidazole in acetonitrile.

The downfield shift at $9.81 \mathrm{ppm}$ of benzimidazolium proton in ${ }^{1} \mathrm{H}$ NMR spectrum of $\mathbf{1 g}$ confirmed its formation which was further supported by the high resolution electrospray mass spectrum (HRMS) which showed a peak at $\mathrm{m} / \mathrm{z} 330.1725$ corresponding to dicationic [M$2 \mathrm{Cl}]^{2+}$ species (calculated mass $\mathrm{m} / \mathrm{z} 330.1727$ ).

The Ag(I)-bis-NHC complex, $\left\{\left[\mathrm{L}\left(\mathrm{L}^{\prime}-\mathrm{NHC}\right)_{2}\right] \mathrm{Ag}\right\} \mathrm{Cl}$ $\left(\mathrm{L}=3,3^{\prime}\right.$-dimethyl-2,2'-dimethoxy-1,1'-binaphthyl, $\mathrm{L}^{\prime}$ $=i$-propyl-benzo[d]imidazole) $(\mathbf{l h})$ was synthesized by metallation of benzimidazolium salt $1 \mathrm{~g}$ with $\mathrm{Ag}_{2} \mathrm{O}$ as the metal precursor in $c a .93 \%$ yield. In particular, $\mathrm{Ag}(\mathrm{I})$-bis-NHC complex (1h) was observed with highly downfield chemical shift at $187.13 \mathrm{ppm}$ in ${ }^{13} \mathrm{C}\left\{{ }^{1} \mathrm{H}\right\}$ NMR spectrum attributed to $\mathrm{Ag}-\mathrm{C}_{\text {carbene }}$ and the HRMS showed peak at 766.2433 corresponding to $[\mathrm{M}-\mathrm{Cl}]^{+}$species (calculated mass $\mathrm{m} / \mathrm{z}$ 766.2432).

The $\left\{\left[\mathrm{L}\left(\mathrm{L}^{\prime}-\mathrm{NHC}\right)_{2}\right] \mathrm{Au}\right\} \mathrm{Cl} \quad\left(\mathrm{L}=3,3^{\prime}\right.$-dimethyl-2,2'dimethoxy-1,1'-binaphthyl, $\mathrm{L}^{\prime}=i$-propyl-benzo[d]imidazole) (1i) complex was obtained by transmetallation of $\mathbf{1 h}$ with the gold precursor $\mathrm{Au}\left(\mathrm{SMe}_{2}\right) \mathrm{Cl}$ in $c a$. $42 \%$ yield. Interestingly, the peak at $177.92 \mathrm{ppm}$ of Au- $\mathrm{C}_{\text {carbene }}$ was observed in its ${ }^{13} \mathrm{C}\left\{{ }^{1} \mathrm{H}\right\}$ NMR spectrum with no peak at $187.13 \mathrm{ppm}$ of $\mathrm{Ag}-\mathrm{C}_{\text {carbene }}$ of its corresponding Ag-bis-NHC (1h) complex. Similarly, HRMS peak was seen at 891.2744 belonging to $[\mathrm{M}-\mathrm{Cl}]^{+}$species (calculated mass $\mathrm{m} / \mathrm{z}$ 891.2735). Furthermore, Ag-bis-NHC (1h) and Au-bis-NHC (1i) complexes were characterized by NMR, IR, mass, elemental analysis and polarimetry. These studies of $\mathrm{Au}(\mathrm{I})-b i s$-NHC complex designated it to be the structural mimic of its corresponding parent $\mathrm{Ag}(\mathrm{I})-$ bis-NHC complex (1h).

As the X-ray structure for these compounds was difficult to obtain even after applying several tricks and methods of crystal growth as well as by changing counter anion. To overcome this problem, we came up with the solution of designing the $\mathbf{1 h}$ and 1i complexes in 'Chemcraft' and to optimize it using GAUSSIAN 09 quantum chemical computations at the B3LYP/SDD, 6-31G(d) level of theory (Figure S25 in Supporting information). In this regard, the atomic coordinates were adopted from two different X-ray structures ${ }^{51}$ CCDC 818680 and CCDC 258234 (Figure S25 in Supporting information), by combining these coordinates the $\left\{\left[\mathrm{L}\left(\mathrm{L}^{\prime}-\mathrm{NHC}\right)_{2}\right] \mathrm{Ag}\right\} \mathrm{Cl}$ (L $=3,3^{\prime}$-dimethyl-2,2'-dimethoxy-1,1'-binaphthyl, $\mathrm{L}^{\prime}=$ $i$-propyl-benzo[d]imidazole) (1h) molecule was constructed using Chemcraft software with hypothetical bond length and bond angles. Finally, the coordinates of this newly designed molecule were fed into the DFT calculations which gave the output of geometry of the optimised structure $\mathbf{1 h}$ (Figure 1) and (Table S1 in Supporting information). A comparison of the adopted solid state crystal structures with that of geometry optimised structure reveals the good conformity in them in terms of measured and calculated bond lengths and bond angles (Table 1). 

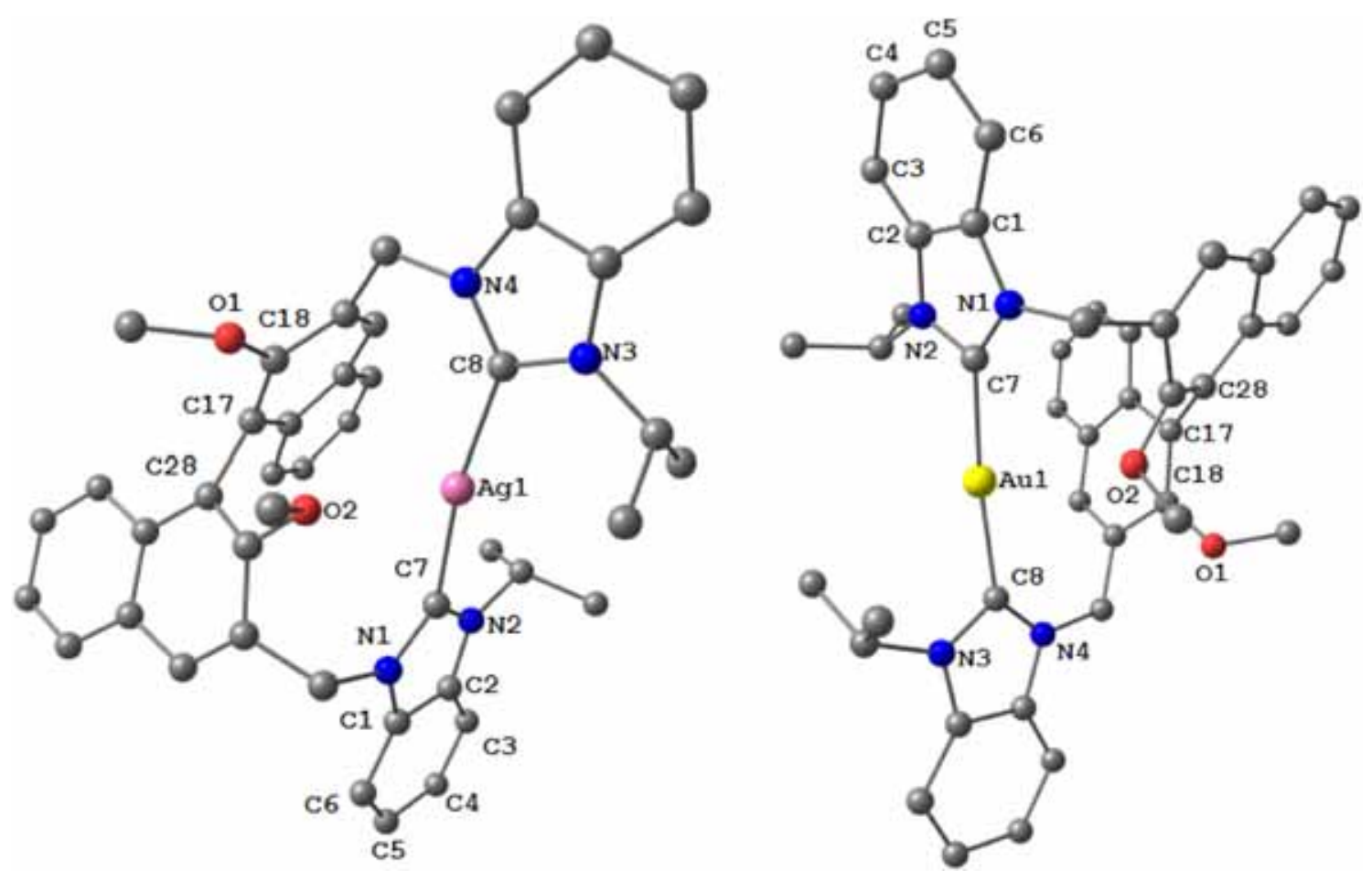

Figure 1. Computed structures of $\mathbf{1 h}$ (left) and $\mathbf{1 i}$ (right) at B3LYP/SDD, 6-31G(d) level of theory.

Table 1. Comparison of X-ray crystal of fragments and computed structures of $\mathbf{1 h}$ and $\mathbf{1 i}$ complexes at B3LYP/SDD, 6-31G (d) level of theory (Supporting information: Figure S25 ${ }^{51}$ ).

\begin{tabular}{|c|c|c|c|c|c|}
\hline \multicolumn{2}{|c|}{$\begin{array}{l}\text { Selected bond lengths } \\
(\AA) \text { and angles }\left(^{\circ}\right) \text { of } \mathbf{1 h}\end{array}$} & \multicolumn{2}{|c|}{$\begin{array}{l}\text { Selected bond lengths } \\
(\AA) \text { and angles }\left({ }^{\circ}\right) \text { of } \mathbf{1 i}\end{array}$} & \multicolumn{2}{|c|}{$\begin{array}{l}\text { Selected bond lengths }(\AA) \text { and } \\
\text { angles }\left({ }^{\circ}\right) \text { of fragments } 51\end{array}$} \\
\hline $\mathrm{C} 7-\mathrm{Ag} 1$ & 2.19421 & $\mathrm{C} 7-\mathrm{Ag} 1$ & 2.210182 & $\mathrm{C} 7-\mathrm{Ag} 1$ & 2.10687 \\
\hline $\mathrm{C} 7-\mathrm{N} 2$ & 1.36275 & $\mathrm{C} 7-\mathrm{N} 2$ & 1.37171 & $\mathrm{C} 7-\mathrm{N} 2$ & 1.35611 \\
\hline $\mathrm{C} 17-\mathrm{C} 28$ & 1.4995 & $\mathrm{C} 17-\mathrm{C} 28$ & 1.50035 & $\mathrm{C} 29-\mathrm{C} 40$ & 1.49989 \\
\hline $\mathrm{C} 7-\mathrm{Ag} 1-\mathrm{C} 8$ & 170.147 & $\mathrm{C} 7-\mathrm{Au} 1-\mathrm{C} 8$ & 173.769 & $\mathrm{C} 7-\mathrm{Ag} 1-\mathrm{C} 12$ & 171.603 \\
\hline $\mathrm{N} 1-\mathrm{C} 7-\mathrm{N} 2$ & 105.856 & $\mathrm{~N} 1-\mathrm{C} 7-\mathrm{N} 2$ & 106.280 & $\mathrm{~N} 1-\mathrm{C} 7-\mathrm{N} 2$ & 105.715 \\
\hline $\mathrm{C} 18-\mathrm{C} 17-\mathrm{C} 28$ & 120.475 & $\mathrm{C} 18-\mathrm{C} 17-\mathrm{C} 28$ & 120.194 & $\mathrm{C} 28-\mathrm{C} 29-\mathrm{C} 40$ & 121.015 \\
\hline
\end{tabular}

The computed structure of $\left\{\left[\mathrm{L}\left(\mathrm{L}^{\prime}-\mathrm{NHC}\right)_{2}\right] \mathrm{Au}\right\} \mathrm{Cl}(\mathrm{L}$ $=3,3^{\prime}$-dimethyl-2,2'-dimethoxy-1,1'-binaphthyl, $\mathrm{L}^{\prime}=$ $i$-propyl-benzo[d]imidazole) (1i) complex (Figure 1) was generated by using the coordinates of geometry optimized structure of its corresponding Ag-bisNHC (1h) complex. These computed structures showed good agreement with the experimental data which was obtained from different spectroscopic and analytical means. The coordinates obtained from the DFT study of Au-bis-NHC complex (1i) are shown in Supporting information (Table S2 in Supplementary Information).

The electronic properties of these complexes further gave an idea that both the silver(I) and gold(I) complexes $\mathbf{1 h}$ and $\mathbf{1 i}$ do not show evidence for metal-metal interactions in their molecular structures. The photoluminescence studies were performed on these complexes in a saturated, glassy solution of $\mathrm{EtOH}: \mathrm{CHCl}_{3}$ (4:1, $\mathrm{v} / \mathrm{v})$ at $77 \mathrm{~K}$ which showed emission peaks at $346 \mathrm{~nm}$ when excited at $255 \mathrm{~nm}$ for $\mathbf{1 g}, \mathbf{1 h}$ and $\mathbf{1 i}$ (E2 in Figure 2). There was no fluorescent peak observed in the emission spectra of $\mathbf{1 h}$ and $\mathbf{1 i}$ complexes, even on excitation at different wavelengths, corresponding to their electronic spectra. Before performing the fluorescence experiments, the absorption spectra were recorded for all these compounds. The UV spectrum of the ligand precursor (1g) showed absorption peaks at 228, 271, 277 and $330 \mathrm{~nm}$. Ag-bis-NHC (1h) and Au-bis-NHC showed (1i) bathochromic shift in their absorption spectra: for $\mathbf{1 h}$ at 246, 276 and $284 \mathrm{~nm}$, and for $\mathbf{1 i}$ at 246 , 


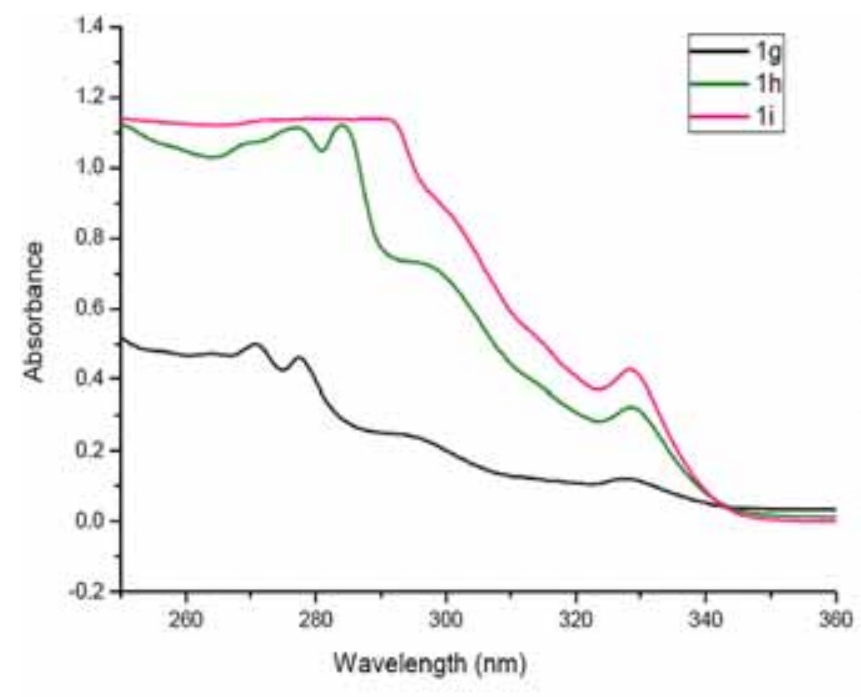

E1

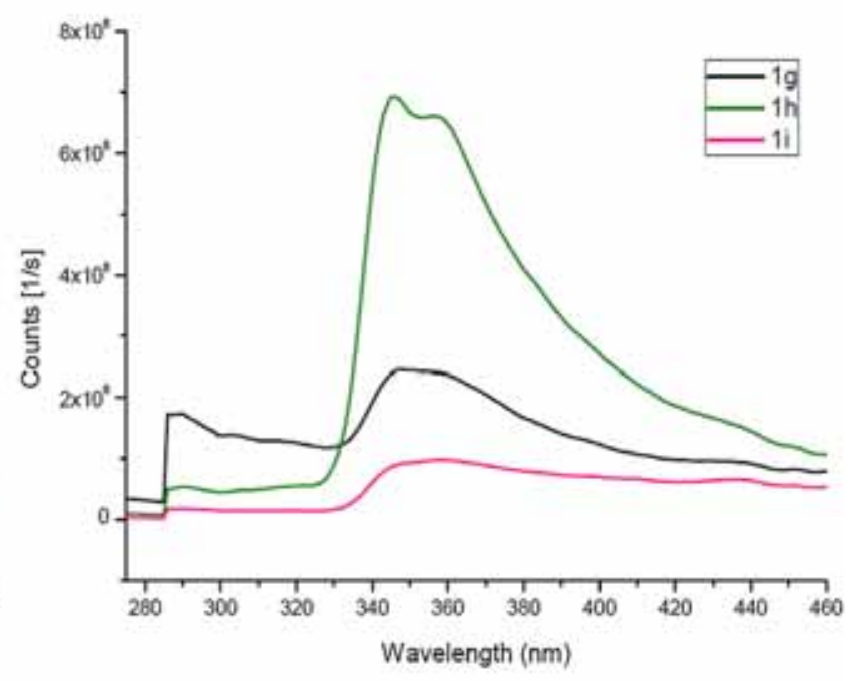

E2

Figure 2. (E1) An overlay plot of UV spectra of ligand $\mathbf{1 g}$ and bis-NHC complexes $\mathbf{1 h}$ and $\mathbf{1 i}$ recorded in $\mathrm{MeOH}$ and $\mathrm{CHCl}_{3}\left(1 \times 10^{-6} \mathrm{M}\right)$, respectively. (E2) An overlay plot of emission spectra of $\mathbf{1}(\mathbf{g}-\mathbf{i})$ measured in a saturated glassy solution of $\mathrm{EtOH}: \mathrm{CHCl}_{3}(4: 1, \mathrm{v} / \mathrm{v})$ at $77 \mathrm{~K}$ upon excitation at $255 \mathrm{~nm}$.

280 and $290 \mathrm{~nm}$, but there was no change in the peak at $330 \mathrm{~nm}$ which is associated with the absorption related to ligand (E1 in Figure 2).

\section{Conclusions}

In summary, $\left\{\left[\mathrm{L}\left(\mathrm{L}^{\prime}-\mathrm{NHC}\right)_{2}\right] \mathrm{M}\right\} \mathrm{Cl}(\mathrm{M}=\mathrm{Ag}$ and $\mathrm{Au})$ type complexes, $\mathbf{1 h}$ and $\mathbf{1 i}$, were synthesized from the bis-N-heterocyclic carbene ligand of $R$-BINOL and benzimidazole framework. The ligand $1 \mathrm{~g}$ and the complexes $\mathbf{1 h}$ and $\mathbf{1 i}$ were characterized by using various spectroscopic and analytical techniques. These studies conspicuously revealed that in both the complexes $\mathbf{1 h}$ and $\mathbf{1} \mathbf{i}$, one metal ion was linearly chelated to a bidentate $\mathrm{N}$-heterocyclic carbene carbons of ligand $\mathbf{1 g}$. In addition, the DFT studies also support the several electronic parameters related to metal-carbene interactions and its orientation with respect to $R$-BINOL scaffold in these $\mathrm{Ag}(\mathrm{I})$ and $\mathrm{Au}(\mathrm{I})$ complexes. Photoluminescence studies were performed on these compounds to check the possibility of metal-metal interactions in these complexes; it gave no proof about such interactions which lend further support to the monometallic molecular structures.

\section{Supplementary Information (SI)}

Spectroscopic and analytical data for $\mathbf{1 g}, \mathbf{1 h}$ and $\mathbf{1 i}$ (Figures S1-S25) and the computational data, B3LYP/SDD, 6-31G(d) level optimized coordinates of the complexes, $\mathbf{1 h}$ and1i (Tables S1 and S2) are available at www.ias.ac.in/chemsci.

\section{Acknowledgements}

Author thanks the Ph.D supervisor Prof. Prasenjit Ghosh for his guidance and support, and IIT Bombay for research fellowship. Author is grateful to the Department of Chemistry, IIT Bombay for Cental Facility and Computational facilities.

\section{References}

1. Yoon T P and Jacobsen E N 2003 Privileged Chiral Catalysts Science 2991691

2. Cesar V Bellemin-Laponnaz, S Gade 2004 Chiral Nheterocyclic carbenes as stereodirecting ligands in asymmetric catalysis Chem. Soc. Rev. 33619

3. Garrison J C and Youngs W J 2005 Ag(I) N-Heterocyclic Carbene Complexes: Synthesis, Structure, and Application Chem. Rev. 1053978

4. Hahn F E and Jahnke M C 2008 Heterocyclic Carbenes: Synthesis and Coordination Chemistry Angew. Chem. Int. Ed. 473122

5. Fortman G C and Nolan S P 2011 N-Heterocyclic carbene (NHC) ligands and palladium in homogeneous cross-coupling catalysis: a perfect union Chem. Soc. Rev. 405151

6. Velazquez H D and Verpoort F 2012 N-heterocyclic carbene transition metal complexes for catalysis in aqueous media Chem. Soc. Rev. 417032

7. Liu W and Gust R 2013 Metal N-heterocyclic carbene complexes as potential antitumor metallodrugs Chem. Soc. Rev. $\mathbf{4 2} 755$

8. Yam V W-W and Lo K K-W 1999 Luminescent polynuclear d10 metal complexes Chem. Soc. Rev. 28323

9. Pugh V J H and Zuo Q S 2001 Optically Active BINOL Core-Based Phenyleneethynylene Dendrimers 
for the Enantioselective Fluorescent Recognition of Amino Alcohols J. Org. Chem. 666136

10. Ray L Shaikh M M and Ghosh P 2008 Shorter Argentophilic Interaction than Aurophilic Interaction in a Pair of Dimeric $(\mathrm{NHC}) \mathrm{MCl}_{2}(\mathrm{M}=\mathrm{Ag}, \mathrm{Au})$ Complexes Supported over a N/O-Functionalized N-Heterocyclic Carbene (NHC) Ligand Inorg. Chem. 47230

11. Liu X Y, Fu X and Zhu Y and Cheng C Y 2011 Novel fluorescent sensor for $\mathrm{Ag}^{+}$and $\mathrm{Hg}^{2+}$ based on the BINOL-pyrene derivative via click reaction Tetrahedron 673181

12. Wang D and Lippard S J 2005 Cellular processing of platinum anticancer drugs Nat. Rev. Drug Discov. 4307

13. Ray S, Mohan R S, Samantaray J K, Shaikh M K, Panda M M and Ghosh P 2007 Anticancer and Antimicrobial Metallopharmaceutical Agents Based on Palladium, Gold, and Silver N-Heterocyclic Carbene Complexes $J$. Am. Chem. Soc. 12915042

14. Jakupec M A, Galanski M A, Hartinger V B, Keppler C $\mathrm{G}$ and Bernhard K 2008 Antitumour metal compounds: more than theme and variations Dalton Trans. 2183

15. Yasukawa T, Miyamura H and Kobayashi S 2014 Chiral metal nanoparticle-catalyzed asymmetric C-C bond formation reactions Chem. Soc. Rev. 431450

16. Chen Y Cheng, Li G, Shelar K, Lu D P and Che W 2014 Phosphorescent polymeric nanomaterials with metallophilic $\mathrm{d} 10 \cdots \mathrm{d} 10$ interactions self-assembled from $\left[\mathrm{Au}(\mathrm{NHC})_{2}\right]^{+}$and $\left[\mathrm{M}(\mathrm{CN})_{2}\right]$ Chem. Sci. 51348

17. Mercs L and Albrecht M 2010 Beyond catalysis: Nheterocyclic carbene complexes as components for medicinal, luminescent, and functional materials applications Chem. Soc. Rev. 391903

18. Glorius F 2007 N-Heterocyclic Carbenes in CatalysisAn Introduction Top. Organomet. Chem. 2113

19. Bourissou D, Guerret O, Gabbai F P and Bertr G 2000 Stable Carbenes Chem. Rev. 10039

20. Arduengo A J, Harlow R L and Kline M 1991 A stable crystalline carbene J. Am. Chem. Soc. 113361

21. Enders D N and Henseler A 2007 Organocatalysis by N-Heterocyclic Carbenes Chem. Rev. 1075606

22. Marion N and Nolan S P 2007 N-Heterocyclic Carbenes as Organocatalysts Angew. Chem. Int. Ed. 462988

23. Visbal R and Gimeno M C 2014 N-heterocyclic carbene metal complexes: photoluminescence and applications Chem. Soc. Rev. $\mathbf{4 3} 3551$

24. Crabtree R H 2005 NHC ligands versus cyclopentadienyls and phosphines as spectator ligands in organometallic catalysis J. Organomet. Chem. 6905451

25. Dove A P, Pratt R C, Lohmeijer B G G, Li H, Hagberg E C, Waymouth R M and Hedrick J L 2006 In $\mathrm{N}$-Heterocyclic Carbenes as Organic Catalysts, in $\mathrm{N}$ Heterocyclic Carbenes in Synthesis S P Nolan (Ed.) (Weinheim, Germany: Wiley-VCH Verlag GmbH \& Co. KGaA) p. 163

26. Kuhl O 2007 The chemistry of functionalised Nheterocyclic carbenes Chem. Soc. Rev. 36592

27. Cui Y Y, Qian Y and Chen G 2012 Luminescent Functional Metal-Organic Frameworks Chem. Rev. 1121126

28. Brendgen T F and Schatz J M 2006 The Suzuki Coupling of Aryl Chlorides in Aqueous Media Catalyzed by in situ Generated Calix[4]arene-Based N-Heterocyclic Carbene Ligands Eur. J. Org. Chem. 20062378
29. Hillier A C, Grasa G A, Viciu M S, Lee H M, Yang C and Nolan S P 2002 Catalytic cross-coupling reactions mediated by palladium/nucleophilic carbene systems $J$. Organomet. Chem. 65369

30. Wang Y M and Toste A D 2014 Development of Catalysts and Ligands for Enantioselective Gold Catalysis Acc. Chem. Res. 47889

31. Normand A T and Cavell K J 2008 Donor-Functionalised N-Heterocyclic Carbene Complexes of Group 9 and 10 Metals in Catalysis: Trends and Directions Eur. J. Inorg. Chem. 20082781

32. Weselinski L S and Jurczak J 2011 The highly enantioselective 1,3-dipolar cycloaddition of alkyl glyoxylatederived nitrones to E-crotonaldehyde catalyzed by hybrid diamines Tetrahedron Lett. 52381

33. Bolm C and Gladysz J A 2003 Introduction: Enantioselective Catalysis. Chem. Rev. 1032761

34. Grill J M, Reibenspies J H and Miller S A 2005 Racemic and chiral expanded salen-type complexes derived from biphenol and binaphthol: Salbip and salbin J. Organomet. Chem. 6903009

35. Shi M and Duan W L 2005 Synthesis of an axially chiral Ir-NHC complex derived from BINAM Appl. Organomet. Chem. 1940

36. Pyykko P 1997 Strong Closed-Shell Interactions in Inorganic Chemistry Chem. Rev. 97597

37. Bauer A and Schmidbaur H 1996 Self-Assembly of $\left[\left(\mathrm{Me}_{2} \mathrm{PhP}\right)_{2} \mathrm{Au}\right]^{+}\left[\mathrm{Au}\left(\mathrm{GeCl}_{3}\right)_{2}\right]^{-}$into Linear Ion Quadruples with an Unusual $[+-+]$ Sequence J. Am. Chem. Soc. 1185324

38. Sluch I M, Miranda A J, Elbjeirami O, Omary M A and Slaughter L M 2012 Interplay of Metallophilic Interactions, $\pi-\pi$ Stacking, and Ligand Substituent Effects in the Structures and Luminescence Properties of Neutral PtII and PdII Aryl Isocyanide Complexes Inorg. Chem. 5110728

39. Yang L, Qin S, Yang X, You F, Hu J, Xie C and Lan J 2010 1,1[prime or minute]-Binaphthyl-based imidazolium chemosensors for highly selective recognition of tryptophan in aqueous solutions Org. Biomol. Chem. 8 339

40. Frisch M J, Trucks G W, Schlegel H B, Scuseria G E, Robb M A, Cheeseman J R, Scalmani G, Barone V, Mennucci B, Petersson G A, Nakatsuji H, Caricato M, Li X, Hratchian H P, Izmaylov A F, Bloino J, Zheng G, Sonnenberg J L, Hada M, Ehara M, Toyota K, Fukuda R, Hasegawa J, Ishida M, Nakajima T, Honda Y, Kitao O, Nakai H, Vreven T, Montgomery Jr J A, Peralta J E, Ogliaro F, Bearpark M, Heyd J J, Brothers E, Kudin K N, Staroverov V N, Kobayashi R, Normand J, Raghavachari K, Rendell A, Burant J C, Iyengar S S, Tomasi J, Cossi M, Rega N, Millam J M, Klene M, Knox J E, Cross J B, Bakken V, Adamo C, Jaramillo J, Gomperts R, Stratmann R E, Yazyev O, Austin A J, Cammi R, Pomelli C, Ochterski J W, Martin R L, Morokuma K, Zakrzewski V G, Voth G A, Salvador P, Dannenberg J J, Dapprich S, Daniels A D, Farkas O, Foresman J B, Ortiz J V, Cioslowski J, Fox D J 2009 Gaussian 09, Revision A.1 (Gaussian, Inc.: Wallingford CT)

41. Becke A D 1988 Density-functional exchange-energy approximation with correct asymptotic behavior Phys. Rev. A: Atom. Mol. Opt. Phys. 383098 
42. Lee C, Yang W and Parr R G 1988 Development of the Colle-Salvetti correlation-energy formula into a functional of the electron density Phys. Rev. B: Condens. Matter 37785

43. Hehre W J, Ditchfield R and Pople J A 1972 SelfConsistent Molecular Orbital Methods. XII. Further Extensions of Gaussian-Type Basis Sets for Use in Molecular Orbital Studies of Organic Molecules $J$. Chem. Phys. 562257

44. Petersson G and Al-Laham M A 1991 A complete basis set model chemistry. II. Open-shell systems and the total energies of the first-row atoms J. Chem. Phys. 94 6081

45. Petersson G A, Bennett A, Tensfeldt $\mathrm{T}, \mathrm{G}$ AlLaham M A, Shirley W A and Mantzaris J 1988 A complete basis set model chemistry. I. The total energies of closed-shell atoms and hydrides of the first-row elements J. Chem. Phys. 89 2193

46. Dolg M, Wedig U, Stoll H and Preuss H 1987 Energyadjusted abinitio pseudopotentials for the first row transition elements J. Chem. Phys. 86866
47. Andrae D H, Dolg U, Stoll and Preub M H 1990 Energy-adjustedab initio pseudopotentials for the second and third row transition elements Theoret. Chim. Acta 77123

48. Alkauskas A, Baratoff A and Bruder C 2004 Gaussian Form of Effective Core Potential and Response Function Basis Set Derived from Troullier-Martins Pseudopotential: Results for Ag and Au J. Phys. Chem. A 1086863

49. Wang X and Andrews L 2001 Gold Hydrides AuH and $\left(\mathrm{H}_{2}\right) \mathrm{AuH}$ and the $\mathrm{AuH}_{3}$ Transition State Stabilized in $\left(\mathrm{H}_{2}\right) \mathrm{AuH}_{3}$ : Infrared Spectra and DFT Calculations $J$. Am. Chem. Soc. 12312899

50. Faza, O N, Alvarez C S and Lera A R 2006 Mechanism of the Gold(I)-Catalyzed Rautenstrauch Rearrangement: A Center-to-Helix-to-Center Chirality Transfer J. Am. Chem. Soc. 1282434

51. Wang L Q, Zhao H, Yao X J, Wang Z Q, Chen Z Q and Wang X G 2012 N-Heterocyclic carbene silver(i), palladium(ii) and mercury(ii) complexes: synthesis, structural studies and catalytic activity CrystEngComm 145330 